TecnoLógicas

ISSN 0123-7799

ISSN-e 2256-5337

Vol. 20, No. 39

Mayo- agosto de 2017

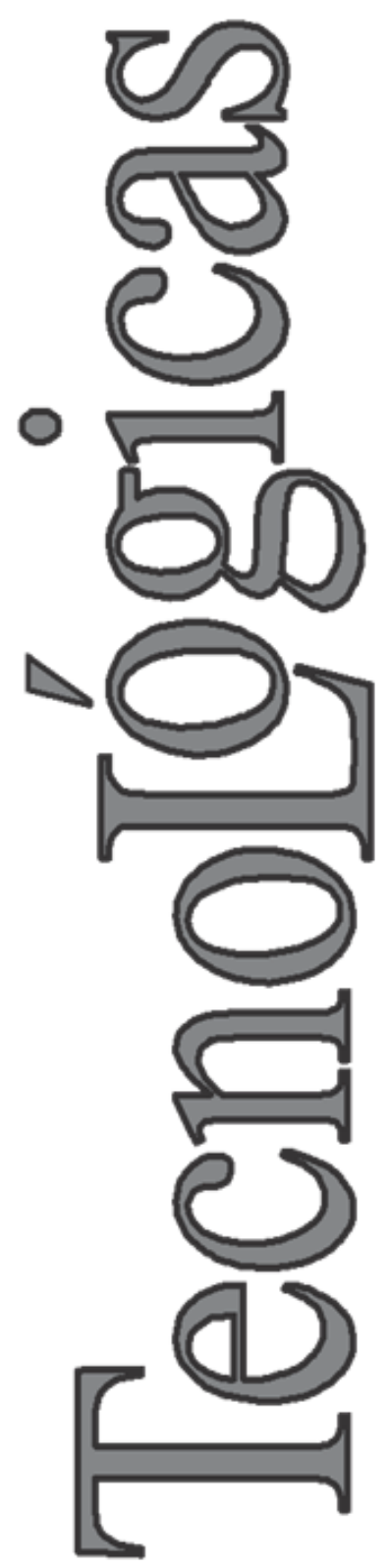

(C) Copyright 2015 por autores y Tecno Lógicas Este trabajo está licenciado bajo una Licencia Internacional Creative Commons Atribución (CC BY)

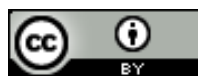

\section{Uso de la perforación dinámica como un método alternativo para determinar el comportamiento mecánico de materiales refractarios}

\section{Dynamic drilling as an alternative method for determining the mechanical behavior of refractories materials}

\author{
Daniel Zuluaga-Castrillón¹, \\ Juan F. Hernández-Ruiz², Fabio Vargas-Galvis ${ }^{3}$, \\ María E. López-Gómez ${ }^{4}$ y \\ Claudia C. Palacio-Espinosa ${ }^{5}$
}

Recibido: 29 de septiembre de 2016, Aceptado: 20 de abril de 2017
Cómo citar / How to cite

D. Zuluaga-Castrillón, J. F. Hernández-Ruiz, F. Vargas-Galvis, M. E. LópezGómez y C. C. Palacio-Espinosa, "Uso de la perforación dinámica como un método alternativo para determinar el comportamiento mecánico de materiales refractarios”, Tecno Lógicas, vol. 20, no. 39, 2017.

1 Ingeniero de Materiales, Departamento de Ingeniería de Materiales, Facultad de Ingeniería, Universidad de Antioquia, MedellínColombia,daniel.zuluagac@udea.edu.co

2 Ingeniero de Materiales, Departamento de Ingeniería de Materiales, Facultad de Ingeniería, Universidad de Antioquia, MedellínColombia, juanf.hernandez@udea.edu.co

$3 \mathrm{PhD}$ en Ciencias, Departamento de Ingeniería de Materiales, Facultad de Ingeniería, Universidad de Antioquia, MedellínColombia, fabio.vargas@udea.edu.co

$4 \mathrm{PhD}$ en Ciencia y Tecnología de Materiales, Departamento de Ingeniería de Materiales, Facultad de Ingeniería, Universidad de Antioquia, Medellín-Colombia, esperanza.lopez@udea.edu.co

$5 \mathrm{PhD}$ en Ciencias, Departamento de Ciencias Físicas, Escuela de Ciencias, Universidad EAFIT, Medellín-Colombia, cpalac12@eafit.edu.co 


\section{Resumen}

La dureza de los materiales cerámicos generalmente se determina mediante microindentación Vickers, para lo cual la muestra debe ser rigurosamente pulida y se deben analizar múltiples huellas para que el resultado sea representativo de las propiedades mecánicas del material, dificultando su uso en el ámbito industrial. En este trabajo se relacionó la dureza Vickers de ladrillos refractarios de alúmina, alúminacircona-sílice (AZS) y de alúmina-cromita, con la resistencia que tienen los mismos a ser perforados por una broca para concreto con punta de carburo de tungsteno. Los refractarios de alúmina y de AZS estudiados fueron fabricados por electrofusión, lo que les confiere alta densificación, mientras que los de alúmina-cromita fueron manufacturados mediante prensado y sinterizado y, por lo tanto, la cohesión entre las partículas que los constituyen y su densificación es baja. La dureza medida para el ladrillo de alúmina y el de AZS es de $1506,00 \pm 99,71 \mathrm{HV}_{1,5 \mathrm{~N}}(15,10 \pm 0,90 \mathrm{GPa})$ y 1028,00 $\pm 95,49 \mathrm{HV}_{2,7 \mathrm{~N}}(10,30 \pm 0,90 \mathrm{GPa})$ respectivamente, mientras que para el de alúminacromita la dureza es sorprendentemente baja, tan solo de $54,00 \pm 2,00 \mathrm{HV}_{50 \mathrm{~N}}(0,54 \pm 0,02$ $\mathrm{GPa}$ ), a pesar de que este material está constituido por partículas altamente duras, lo cual es producto de la baja cohesión de dichas partículas. Los resultados obtenidos indican que existe buena concordancia entre la resistencia a la perforación y la dureza Vickers de los ladrillos estudiados, por lo que el ensayo de perforación mecánica puede ser utilizado para predecir la dureza tanto en materiales cerámicos bien densificados, como en aquellos poco densificados.

\section{Palabras clave}

Refractarios cerámicos, propiedades mecánicas, resistencia a la perforación, microindentación.

\section{Abstract}

Ceramic materials hardness is usually determined by Vickers microindentation, which requires rigorous sample preparation and highly demanding analysis of traces turning this method difficult to use in industrial applications. This research is focused to correlate Vickers Hardness to drilling resistance by a hard-tipped drill-bit on alumina, alumina-zirconia-silica (AZS) and alumina-chromite refractories bricks. Alumina and AZS bricks were manufactured by electromelting process leading to obtain highly densified microstructures, while alumina-chromite bricks were manufactured by pressing and sintering which conducts to lower densification and particles cohesion within the bricks microstructure. Alumina and AZS refractories measured hardness is $1506.00 \pm 99.71 \mathrm{HV}_{1,5 \mathrm{~N}}(15.10 \pm 0.90 \mathrm{GPa})$ and $1028.00 \pm 95.49 \mathrm{HV}_{2,7 \mathrm{~N}}(10.30 \pm 0.90 \mathrm{GPa})$ respectively, which is in contrast with the low measured hardness on alumina-chromite bricks of $54.00 \pm 2.00 \mathrm{HV}_{50 \mathrm{~N}}(0.54 \pm 0.02 \mathrm{GPa})$ despite of the high hardness of alumina and chromite used as feedstock maybe due to a low cohesion between the particles. Drilling resistance results showed a well-fitted behavior regarding the measured Vickers hardness then the drilling resistance test is useful to predict the hardness of highly densified as well as of friable ceramic materials.

\section{Keywords}

Ceramic refractories, mechanical properties, dynamic drilling, micro hardness. 


\section{INTRODUCCIÓN}

En los últimos años el uso de los materiales cerámicos en diferentes campos de la industria ha crecido sustancialmente y con ello la necesidad de evaluar sus propiedades mecánicas, con el fin de predecir su comportamiento o determinar si ellos podrían operar o seguir operando bajo determinadas condiciones [1]-[3].

La dureza es una de las propiedades más relevantes en la mayoría de los materiales cerámicos, y, por lo tanto, la búsqueda de métodos de medida de esta propiedad ha motivado la realización de múltiples estudios.

En la actualidad la indentación a escalas macro, micro y nano, utilizando indentadores de tipo Vickers, Knoop y Berkovich, constituye el conjunto de técnicas más utilizadas para determinar la dureza de materiales cerámicos a partir de la medida de la relación entre la carga aplicada por el indentador y el área de la huella residual o del área proyectada en el material a causa de la indentación [4]-[7]. Los indentadores empleados para la medida de la dureza de materiales cerámicos deben ser lo suficientemente duros y rígidos para poder penetrar en el material sin deformarse ni deteriorarse y además su punta debe ser lo suficientemente aguda para producir en el cerámico una deformación plástica medible sin generar agrietamiento excesivo en él.

Lo anterior implica que cualquiera que sea la escala de medición, el área de material afectado por una indentación es muy pequeña respecto a su volumen, por lo que el resultado de la medida es muy localizada, dificultando la determinación de la dureza global de materiales estructuralmente heterogéneos [8], [9], entendiendo como cerámicos estructuralmente heterogéneos aquellos que tienen demasiados defectos como poros o grietas. Con el fin de facilitar la medida de la dureza en cerámicos con alto contenido de defectos estructurales, se ha incrementado la escala de la indentación respecto al tamaño de los poros o de los defectos presentes en su estructura; no obstante, esto puede conllevar a fallas en el material, tales como: descascaramiento, colapso o densificación, las cuales modifican la respuesta elasto-plástica del material de la cual dependen las medidas de su dureza [10]. Así mismo, algunas herramientas estadísticas y matemáticas han sido propuestas para minimizar el efecto de la heterogeneidad del material sobre la medida de su dureza [11]-[13].

Otra de las desventajas aparentes que tiene la determinación de la dureza mediante indentación, es que la zona sobre la que se realiza la medición debe ser lo suficientemente lisa para evitar alteraciones en la medida a causa de la superficie irregular que pueda tener la muestra, lo cual en la mayoría de los casos implica la ejecución de procesos de preparación que demandan tiempos prolongados y que en ocasiones hacen de este un método de medida destructivo, por la necesidad de cortar y pulir el material o la pieza a evaluar, lo que en ocasiones dificulta su uso a nivel industrial [14], [15].

Como respuesta a la búsqueda de alternativas para la medida de las propiedades mecánicas de materiales heterogéneos se pueden citar los resultados obtenidos por Julius Hirschwald, quien, en 1908 determinó la relación entre la resistencia a la perforación de rocas y arcillas, tanto en seco, como en húmedo, calculando el "coeficiente de ablandamiento", a partir de las revoluciones necesarias para obtener una misma profundidad de perforación, utilizando para ello cuchillas con filo liso y tipo sierra [16].

Por su parte, en 1963 el IBP (Institute of Building Physics), construyó un prototipo para la medida de la resistencia a la perforación en rocas, el cual evolucionó a la versión portátil que el mismo instituto desarrolló a principios de los años 90 [16].

En 1992, Alfes y sus colaboradores correlacionaron la resistencia a la perforación de rocas con su dureza obteniendo 
resultados concordantes lo que les permitió concluir que estas correlaciones son útiles para medir la degradación en rocas. Estos resultados fueron complementados por [17], [18] y publicados en 2004.

En 1996 inició el "European EC Hardrock Project", cuyo objetivo fue desarrollar un nuevo equipo de perforación y establecer los lineamientos de un método estandarizado, como resultado de lo cual se dispone en la actualidad de equipos que pueden incluso realizar mediciones de torque. En consecuencia, en 2001 y 2004 la compañía italiana Sint Technology y la alemana Geotron-Elektronik desarrollaron nuevos dispositivos [16].

Actualmente, se dispone de un equipo conocido como DRMS (Drilling Resistance Measurment System), el cual mide el empuje de la broca utilizada para la perforación en función de la profundidad perforada. El proceso inicia con el contacto no dinámico entre la broca y la superficie del material a evaluar, generando una indentación en él, seguido de un contacto dinámico por rotación de la broca durante el cual el material es perforado [16], [19]. Los trabajos descritos han permitido tomar medidas oportunas y/o preventivas para la conservación de monumentos arquitectónicos construidos en piedra, e incluso hoy en día es aceptado el método de perforación como una alternativa para medir la dureza en algunos minerales como el corindón [16], [20].

Así mismo, [21], determinaron la relación existente entre el diámetro de la broca y la carga aplicada con la profundidad de perforación de ensayos dinámicos, al mismo tiempo que relacionaron la resistencia a la penetración bajo contacto estático con la dureza de recubrimientos del sistema $\mathrm{Al}_{2} \mathrm{O}_{3}-\mathrm{TiO}_{2}$ elaborados por proyección térmica por plasma atmosférico. Los resultados obtenidos les permitieron establecer que el ensayo de perforación puede ser útil para adquirir información sobre la dureza y el módulo elástico, debido a los esfuerzos de cizalla aplicados por la broca sobre la estructura lamelar del recubrimiento y que un análisis similar podría ser llevado a cabo en cerámicos en volumen heterogéneos debido a la similitud de las características estructurales de estos materiales y las de los recubrimientos evaluados [21]. Por su parte [22], [23], evaluaron el efecto del afilado de brocas de acero rápido (HSS), sobre la resistencia a la perforación de recubrimientos cerámicos depositados por proyección térmica, encontrando que éstas se pueden reafilar sin que pierdan sus propiedades mecánicas [22], [23].

En este trabajo se evaluó la correlación existente entre la microdureza Vickers y la resistencia a la perforación de dos ladrillos refractarios electrofundidos y uno fabricado mediante prensado y sinterizado, utilizando una broca para perforación de concreto, cuya punta es fabricada en carburo de tungsteno.

\section{METOdOLOGíA}

\subsection{Preparación de las muestras}

Con el fin de relacionar la resistencia a la perforación de cerámicos heterogéneos con su dureza Vickers, se analizaron 3 refractarios diferentes: dos de ellos de alúmina y alúmina-circona-sílice (AZS) fabricados por electrofusión y el otro de alúmina-cromita fabricado por prensado y sinterizado. La resistencia a la perforación de estos materiales fue evaluada sobre su superficie, tanto pulida como sin pulir para establecer si existe algún efecto del acabado superficial sobre su resistencia a la penetración por una broca para concreto con punta de carburo de tungsteno. La superficie de las muestras fue desbastada de manera manual realizando movimientos unidireccionales con papel abrasivo de $\mathrm{SiC}$ de diferentes granos con una secuencia de P320, P400, P600, P1000 y P2000; posteriormente fueron pulidas con movimientos circulares utilizando como abrasivo pasta de diamante de 3 y $1 \mu \mathrm{m}$ respectivamente, evi- 
tando el desprendimiento excesivo de material que pudiera afectar las medidas de las propiedades mecánicas.

\subsection{Análisis químico, de fases y de la estruc- tura de los materiales}

La composición química de los refractarios fue determinada mediante Fluorescencia de Rayos X (FRX) por longitud de onda dispersiva utilizando un equipo marca Thermo de referencia comercial ARLOptim'x, mientras que los elementos que constituyen la punta de la broca se detectaron mediante el sistema de análisis de energía dispersiva de Rayos X acoplado al Microscopio Electrónico de Barrido (MEB) Jeol JSM 6490 LV.

Por su parte, se cuantificó la porosidad y se analizaron las fases cristalinas presentes en los ladrillos refractarios, con el fin de estimar su dureza. La porosidad fue medida mediante análisis de imágenes de acuerdo con el procedimiento establecido por [24] utilizando el programa Image J, a partir de fotografías tomadas mediante el MEB. Por su parte, el ensayo de Difracción de Rayos X (DRX) se llevó a cabo empleando el equipo PANalytical de referencia EMPYREANTM con una velocidad de barrido de $0,050^{\circ} / \mathrm{min}$ y un rango de detección $2 \theta$ desde $10^{\circ}$ hasta $80^{\circ}$. Para identificar las fases se utilizó el programa High-Score Plus y su cuantificación se hizo mediante el método de Rietveld.

\subsection{Caracterización mecánica por microin- dentación}

La caracterización mecánica tanto de la punta de la broca como de los refractarios se realizó en un microindentador Shimadzu HMV-G series ${ }^{\mathrm{TM}}$, siguiendo los parámetros establecidos en la norma ASTM C1327. Las indentaciones se realizaron sobre las muestras pulidas por exigencia del tipo de ensayo, ya que este tipo de prueba no debe ser realizada sobre muestras de superficie rugosa como la de los refractarios sin pulir. Se empleó un indentador Vickers aplicando sobre él una carga de $25,50,100,150,200,270,300$ y 500 g, realizando sobre cada muestra 20 indentaciones para cada carga, con el fin de establecer la carga a la cual el valor medido es representativo de la dureza de cada material, tal y como lo establece la norma mencionada. Además, para el ladrillo de alúmina-cromita se realizaron indentaciones a una carga de 5000 gramos con el fin de que las huellas tuviesen un tamaño superior al de los defectos presentes en la estructura de este material y poder estudiar el efecto del tamaño de la indentación sobre sus propiedades mecánicas.

\subsection{Pruebas de perforación}

Para todos los procedimientos de perforación se utilizó un taladro de banco equipado con un sistema electromecánico capaz de registrar en tiempo real la carga aplicada a la muestra y provisto de un comparador de carátula Miyutoyo ID-C125B, cuya sensibilidad es de $1 \mu \mathrm{m}$, con el objetivo de evaluar la profundidad de perforación a lo largo del ensayo. Los ensayos se realizaron a través de pruebas dinámicas utilizando una broca de $8,00 \mathrm{~mm}$ de diámetro para concreto, marca DeWalt ${ }^{\mathrm{TM}}$. La carga ejercida por la broca fue de $290 \mathrm{~N}$ para todos los ensayos; a la vez que la velocidad de rotación fue de $360 \mathrm{rpm}$ y el tiempo de perforación fue de $180 \mathrm{~s}$. Se realizaron 10 perforaciones sobre cada una de las muestras pulidas y sin pulir. Se empleó un flujo de aire constante en la zona de contacto entre la broca y la muestra a evaluar con el objetivo de eliminar el material que pueda desprenderse durante el ensayo y así reducir el efecto de estos residuos en los resultados obtenidos. La superficie perforada de los refractarios fue analizada mediante MEB y el perfil de profundidad de cada hueco fue determinado haciendo un barrido con un comparador de carátula similar al incorporado al taladro de banco. Dado que durante cada uno de los ensayos la superfi- 
cie de las brocas experimenta un leve desgaste, se realizó un reafilado de su punta después de cada una de las pruebas, utilizando el equipo Doctor Drill 750X, a un ángulo de $135^{\circ}$ hasta que su geometría y acabado fuese idéntico al que tenían antes de ser utilizadas.

\section{RESULTADOS Y DISCUSIÓN}

\subsection{Análisis químico, de fases y de la poro- sidad de los materiales}

Los resultados del análisis químico realizado mediante FRX indican que el ladrillo que ha sido denominado como refractario de alúmina, además de $\mathrm{Al}_{2} \mathrm{O}_{3}$ contiene cantidades importantes de sílice $\left(\mathrm{SiO}_{2}\right)$, circonia $\left(\mathrm{ZrO}_{2}\right)$, óxido de calcio $(\mathrm{CaO}) \mathrm{y}$ óxido de magnesio $(\mathrm{MgO})$. Por su parte, el ladrillo denominado como AZS está constituido principalmente por alúmina, circona y sílice, mientras que el de alúminacromita además contiene un porcentaje importante de sílice, tal y como se muestra en la Tabla 1.
Por su parte, el análisis elemental realizado a la punta de la broca permitió establecer que este material está constituido principalmente por tungsteno, cobalto, hierro y carbono, con pequeñas trazas de cobre, los cuales son los constituyentes característicos de la punta de las brocas utilizadas para perforar concreto [25].

El análisis de imágenes realizado indica que la porosidad de los refractarios de alúmina, de AZS y del de alúmina-cromita es $1,80 \%, 4,50 \%$ y $12,10 \%$ en área respectivamente.

Por su parte, los resultados de la prueba de DRX realizada al refractario de alúmina indican que las fases que constituyen este material son $\mathrm{Al}_{2} \mathrm{O}_{3}-\alpha, \quad \mathrm{ZrO}_{2}-\mathrm{B}$, $\mathrm{MgAl}_{2} \mathrm{O}_{4}-\sigma$, y $\mathrm{SiO}_{2}$ (cuarzo). Mientras que en el refractario AZS las fases cristalinas identificadas fueron $\mathrm{Al}_{2} \mathrm{O}_{3}-\alpha, \mathrm{ZrO}_{2}-B, \mathrm{ZrO}_{2}-\tau$ y $\mathrm{SiO}_{2}$ (cristobalita) y en el refractario de alúmina-cromita fueron $\mathrm{Al}_{2} \mathrm{O}_{3}-\mathrm{a}, \quad \mathrm{Cr}_{2} \mathrm{O}_{3}$ (eskolaite) y $\mathrm{SiO}_{2}$ (cristobalita). Los porcentajes en peso de cada una de estas fases en los refractarios y su dureza son mostrados en la Tabla 2. En las Fig. 1, 2 y 3 se presentan los difractogramas de rayos $\mathrm{X}$ de los refractarios analizados.

Tabla 1. Resultados del análisis químico realizado a los refractarios. Fuente: Autores.

\begin{tabular}{|c|c|c|c|c|c|c|}
\hline Refractario & \multicolumn{2}{|c|}{$\mathrm{Al}_{2} \mathrm{O}_{3}$} & \multicolumn{2}{|c|}{ AZS } & \multicolumn{2}{|c|}{ Alúmina-cromita } \\
\hline Óxido & Peso (\%) & $\begin{array}{c}\text { Desviación } \\
\text { estándar }\end{array}$ & Peso (\%) & $\begin{array}{c}\text { Desviación } \\
\text { estándar }\end{array}$ & Peso (\%) & $\begin{array}{c}\text { Desviación } \\
\text { estándar }\end{array}$ \\
\hline $\mathrm{Al}_{2} \mathrm{O}_{3}$ & 83,75 & 0,18 & 46,76 & 0,23 & 67,50 & - \\
\hline $\mathrm{SiO}_{2}$ & 7,87 & 0,12 & 11,83 & 0,20 & 4,00 & - \\
\hline $\mathrm{ZrO}_{2}$ & 3,13 & 0,11 & 39,82 & 0,14 & - & - \\
\hline $\mathrm{CaO}$ & 2,74 & 0,08 & 0,57 & 0,06 & 0,11 & - \\
\hline $\mathrm{MgO}$ & 2,10 & 0,07 & - & - & - & - \\
\hline $\mathrm{Fe}_{2} \mathrm{O}_{3}$ & 0,14 & 0,01 & 0,21 & 0,01 & 0,15 & - \\
\hline $\mathrm{Cr}_{2} \mathrm{O}_{3}$ & - & - & - & - & 25,50 & - \\
\hline Otros* & 0,27 & & 0,81 & & 2,74 & - \\
\hline
\end{tabular}




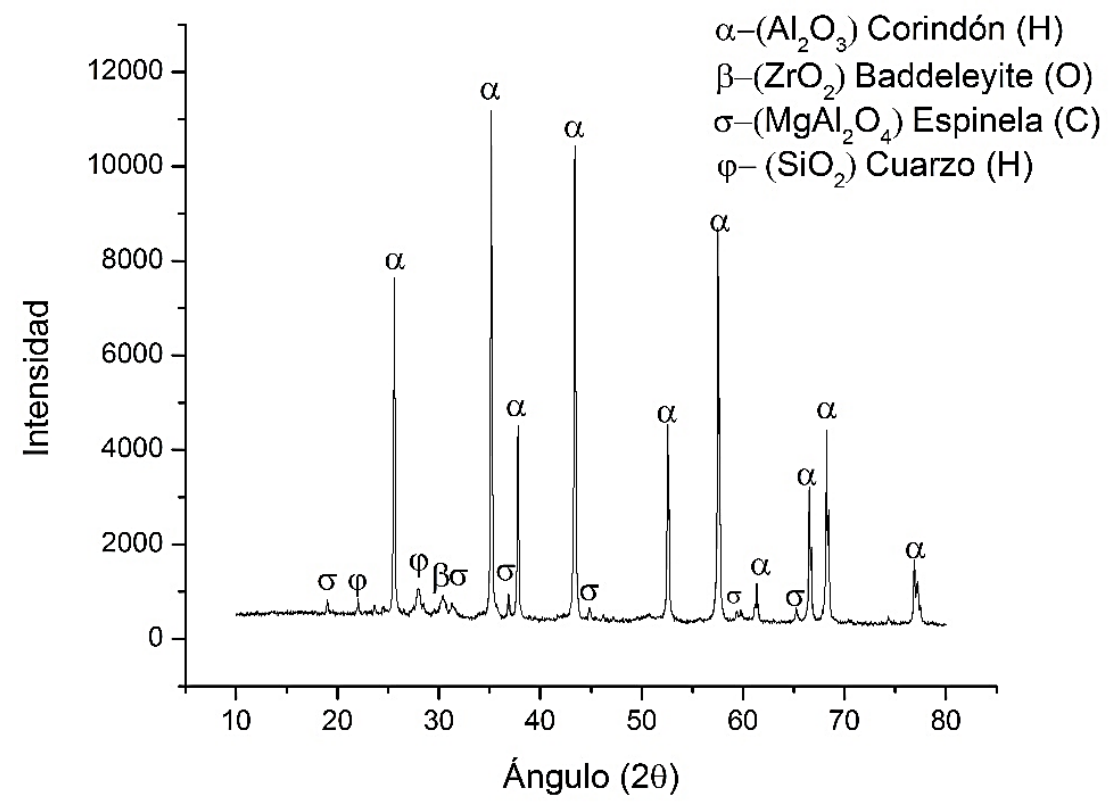

Fig. 1. Espectro de difracción de rayos X para la muestra de alúmina electrofundida. Fuente: Autores.

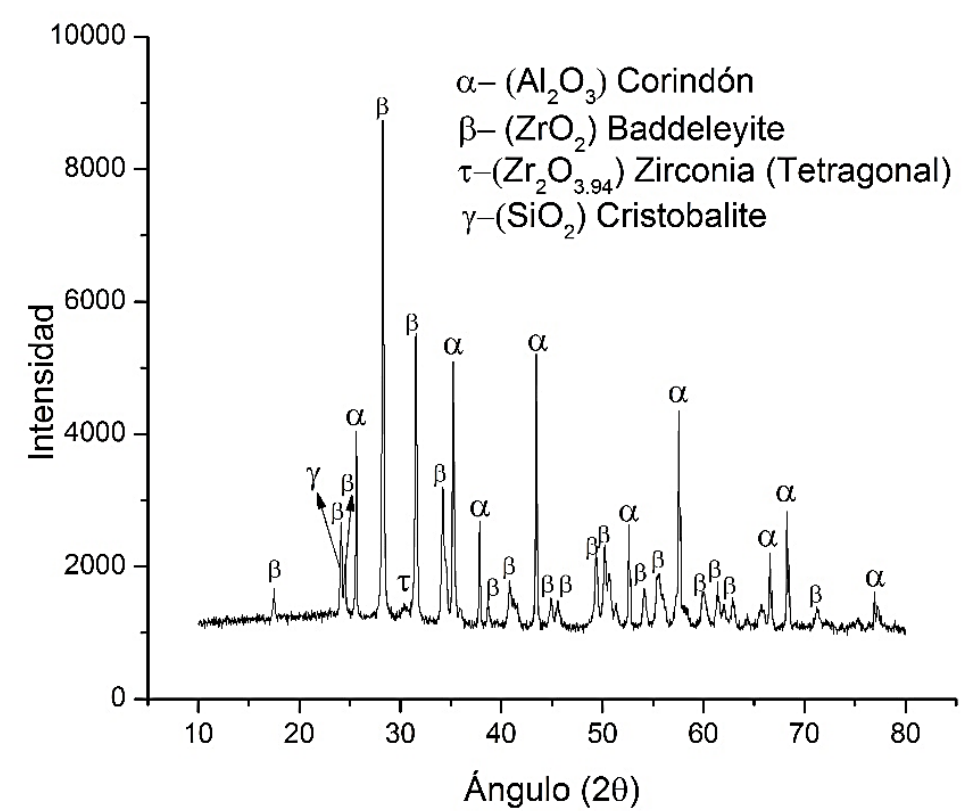

Fig. 2. Espectro de difracción de rayos X para la muestra de AZS electrofundida. Fuente: Autores. 
Uso de la perforación dinámica como un método alternativo para determinar el comportamiento mecánico de materiales refractarios

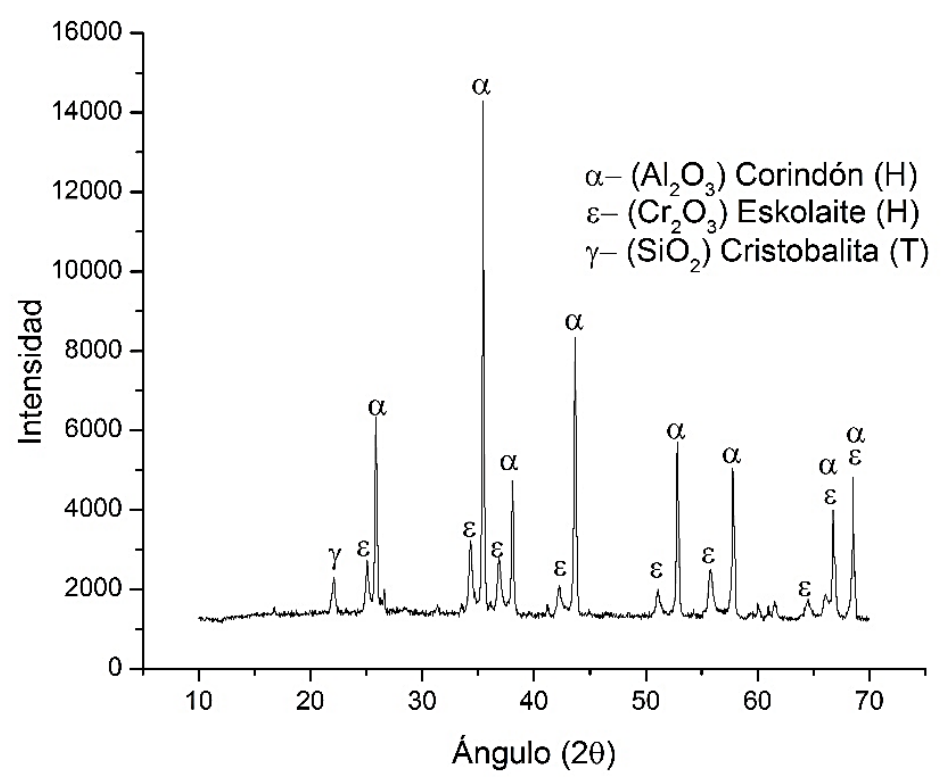

Fig. 3. Espectro de difracción de rayos X para la muestra de alúmina-cromita prensada y sinterizada. Fuente: Autores.

Tabla 2. Porcentaje de fases en cada recubrimiento y su respectiva dureza Vickers [7], [26]-[30]. Fuente: Autores.

\begin{tabular}{llcc}
\hline Refractario & \multicolumn{2}{c}{$\begin{array}{c}\text { Fases } \\
\% \text { en peso }\end{array}$} & $\begin{array}{c}\text { Dureza } \\
\mathrm{HV}[\mathrm{GPa}]^{*}\end{array}$ \\
\hline \multirow{4}{*}{ Alúmina } & $\mathrm{Al}_{2} \mathrm{O}_{3}-\mathrm{a}$ & 95,60 & 16,00 \\
& $\mathrm{ZrO}_{2}-6$ & 0,50 & 12,00 \\
& $\mathrm{MgAl}_{2} \mathrm{O}_{4}-\sigma$ & 2,70 & 13,00 \\
& $\mathrm{SiO}_{2}$ (cuarzo) & 1,20 & 11,00 \\
\hline \multirow{4}{*}{ AZS } & $\mathrm{Al}_{2} \mathrm{O}_{3}-\mathrm{a}$ & 62,39 & 16,00 \\
& $\mathrm{ZrO}_{2}-6$ & 35,47 & 12,00 \\
& $\mathrm{ZrO}_{2}-\tau$ & 0,48 & 11,50 \\
& $\mathrm{SiO}_{2}$ (cristobalita) & 1,65 & 11,00 \\
\hline \multirow{4}{*}{ Alúmina-cromita } & $\mathrm{Al}_{2} \mathrm{O}_{3}$ - $\mathrm{a}$ & 83,10 & 16,00 \\
& $\mathrm{Cr}_{2} \mathrm{O}_{3}$ (eskolaite) & 16,80 & 24,00 \\
& $\mathrm{SiO}_{2}$ (cristobalita) & 0,10 & 11,00 \\
\hline
\end{tabular}

* Valores reportados por otros autores, algunos de ellos no especifican la carga utilizada. 


\subsection{Propiedades mecánicas}

En la Fig. 4, se muestra la curva de calibración utilizada para determinar la dureza real de los refractarios de alúmina y del de AZS. En esta figura, se puede apreciar que en cada uno de estos materiales se presenta una zona de sobreestimación de la dureza (a bajas cargas aplicadas sobre la muestra), lo cual se debe a la recuperación elástica excesiva que generalmente tienen los materiales cerámicos y que hacen que una vez retirada la carga las huellas sean más pequeñas de lo que realmente deberían ser de acuerdo con la dureza del material. Por otra parte, se puede apreciar también una zona de subestimación de la dureza, que se da cuando la carga aplicada sobre la muestra es excesiva y se presenta a causa de las fracturas que se producen en el material y que reducen su resistencia a la penetración del indentador. Este comportamiento es muy común en los materiales cerámicos, dada su alta fragilidad. A partir de la curva de calibración se determinó el valor de la carga crítica que corresponde a la zona donde se estabiliza el valor de dureza o zona en la cual la dureza se mantiene casi invariante conforme se varía la carga. Para el caso de las muestras de refractario de alúmina la carga crítica está entre 100 y 150 gf y para las AZS entre 250 y 300 gf, lo cual corresponde a valores de micro dureza de $1506,00 \pm 99,71 \mathrm{HV}_{1,5 \mathrm{~N}}(15,10 \pm 0,90$ $\mathrm{GPa})$ y de $1028,00 \pm 95,49 \mathrm{HV}_{2,7 \mathrm{~N}}(10,30 \pm$ $0,90 \mathrm{GPa})$ respectivamente. Este valor es cercano al encontrado en la literatura para materiales similares y cuya dureza varía entre 14 y $16 \mathrm{GPa}$ para el caso de la alúmina y entre 9 y $10 \mathrm{GPa}$ para el caso del AZS [14], [15], [31].

En el refractario de alúmina-cromita no fue posible determinar la micro-dureza
Vickers, dado que la cohesión entre las partículas que lo constituyen es muy baja $\mathrm{y}$, por lo tanto, las huellas obtenidas después de aplicada la carga no cumplen con los criterios de aceptación y rechazo especificados en la norma ASTM C1327, ver Fig. 5. Sin embargo, sobre esta muestra se realizaron indentaciones aplicando una carga de 5000 gf para producir huellas con un tamaño superior al de los defectos presentes en la microestructura y así reducir el efecto de la interfaz entre partículas, obteniendo como resultado una dureza Vickers de 54,00 $\pm 2,00 \mathrm{HV}_{50 \mathrm{~N}}(0,54 \pm 0,02$ GPa).

Adicionalmente, utilizando (1) [13] se estimó la dureza de los refractarios estudiados a partir de los porcentajes de fases que los constituyen, ver Tabla 2 y los porcentajes de porosidad determinados por análisis de imágenes encontrando que la dureza estimada para el refractario de alúmina es 15,27 $\mathrm{GPa}$, mientras que la del AZS y del de alúmina-cromita es de 13,17 y 13,07 GPa respectivamente.

$$
H V * *=\sum \frac{X_{i}}{100} H V_{i}\left(1-\frac{2 X_{p}}{100}\right)
$$

De donde $\mathrm{HV}^{* *}$ es la microdureza $\mathrm{Vi}$ ckers estimada, $\mathrm{HV}_{\mathrm{i}}$ es la microdureza para cada uno de los constituyentes, $\mathrm{X}_{\mathrm{i}}$ es el porcentaje en peso de cada una de las fases del material y $\mathrm{X}_{\mathrm{p}}$ el porcentaje de poros de cada muestra.

De otra parte, los resultados de las pruebas de indentación realizadas a la punta de la broca de mampostería indican que su microdureza Vickers es de 1386,44 $\pm 71,10 \mathrm{HV}_{3 \mathrm{~N}}(13,60 \pm 0,70 \mathrm{GPa})$ y fue medida aplicando 300 gf de carga durante 15 s. 
Uso de la perforación dinámica como un método alternativo para determinar el comportamiento mecánico de materiales refractarios
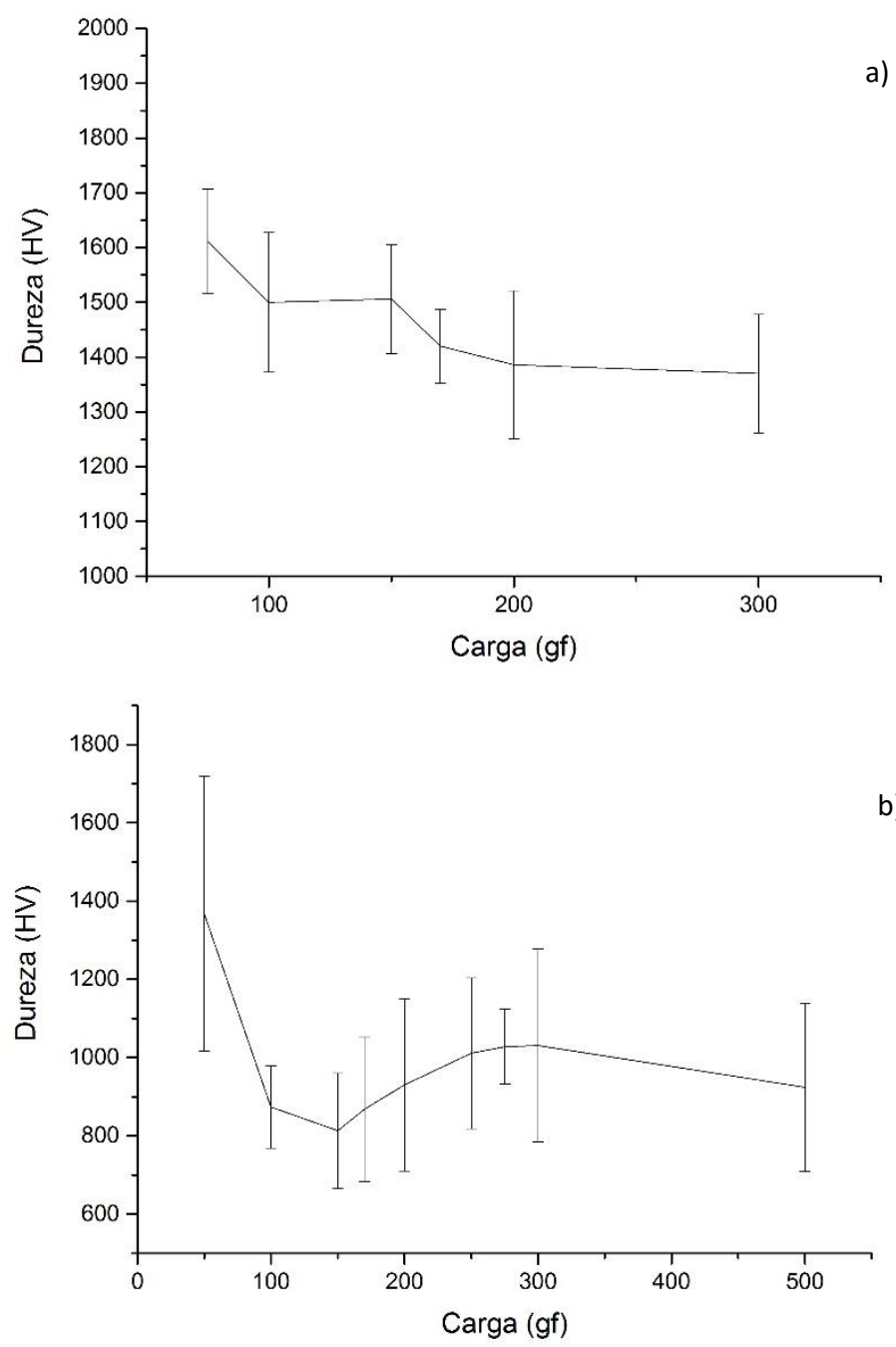

Fig. 4. Curvas de calibración de la dureza para los refractarios de: a) alúmina b) AZS. Fuente: Autores.

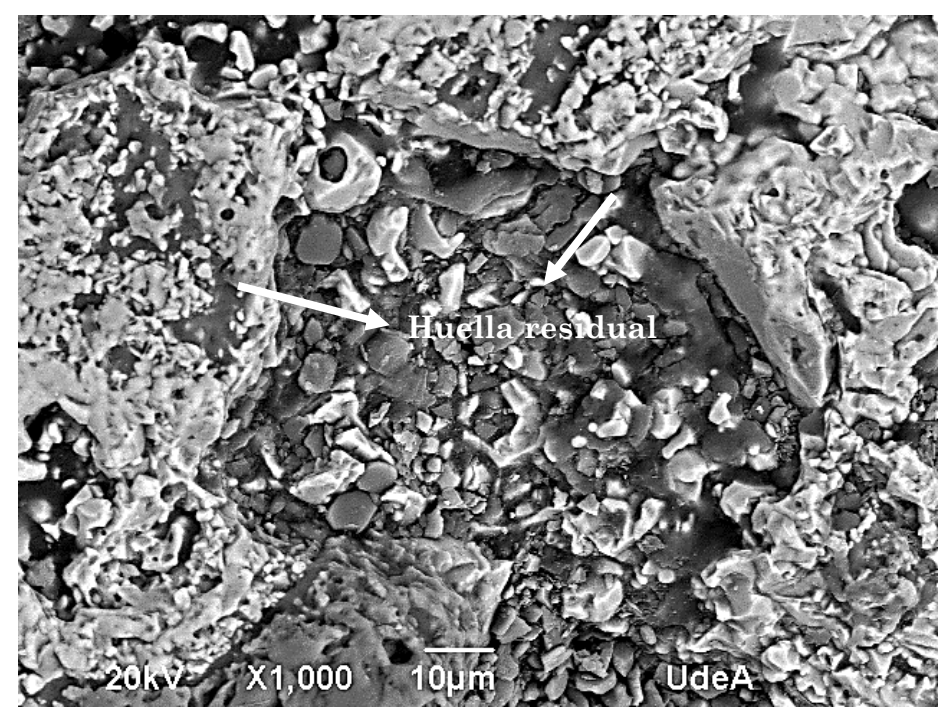

Fig. 5. Huella residual producida por el indentador Vickers en un refractario de alúmina-cromita. Fuente: Autores. 


\subsection{Pruebas de perforación}

Los resultados de las pruebas de perforación realizadas, tanto a los ladrillos cuya superficie fue pulida, como a aquellos sin pulir se pueden observar en la Fig. 6, la cual, describe la profundidad de perforación determinada en todo instante por el comparador de carátula en función del tiempo del ensayo. Las curvas presentadas indican que la profundidad aparentemente perforada crece rápidamente durante los 10 primeros segundos y posteriormente se estabiliza, ver Fig. 6. Cabe señalar que, aunque la profundidad perforada se estabiliza durante el ensayo, su magnitud puede verse ligeramente afectada por diferentes fenómenos, como las deformaciones elásticas presentadas tanto por la broca como por el material perforado y el desgaste de la broca, produciendo ligeras fluctuaciones alrededor de la profundidad de perforación, como se evidencia en las gráficas de la Fig. 6 , luego de $10 \mathrm{~s}$ de ensayo. En las pruebas realizadas sobre los ladrillos pulidos esta profundidad aparentemente perforada es inversa a la dureza real del refractario, lo que indica que esta propiedad mecánica del material es relevante y se ve reflejada en su resistencia a ser perforado. Sin embargo, sobre los ladrillos no pulidos no existen cambios evidentes de dicha profundidad, dado que las asperezas de la superficie podrían conllevar a fracturas del material que no dependen directamente de su dureza.

La profundidad de perforación medida en tiempo real es considerada aparente debido a que las partículas desprendidas, principalmente desde el material perforado colmatan parcialmente el hueco producido y por lo tanto reducen la profundidad medida. Esta afectación es mayor en aquellos materiales menos resistentes a la perforación, en los que la cantidad de partículas desprendidas es mayor.

En la Fig.7 se muestran imágenes de la morfología de las zonas perforadas para cada uno de los materiales, y se puede observar que para el refractario de alúmina se encuentra un hueco de perforación bien definido, sin evidenciar presencia de fenómenos de deslizamiento de la broca $u$ otro tipo de defectos generados durante el ensayo (ver Fig.7.a), lo cual contrasta con los refractarios de AZS y alúmina-cromita en los que se presenta, para el caso de la muestra AZS un orificio de forma irregular que sugiere que durante el proceso de perforación hubo pérdidas momentáneas de contacto entre la punta de la broca y el material perforado (ver Fig.7.b), y para el caso de la muestra de alúmina-cromita cráteres formados al interior del hueco perforado a causa de la baja cohesión entre las partículas que lo conforman (ver Fig.7.c).

Teniendo en cuenta lo anterior, además de la profundidad medida en tiempo real, se determinó la profundidad máxima perforada de cada hueco después de finalizados los ensayos, como se presenta en la Tabla 3.

La profundidad máxima perforada en cada material se correlacionó con el respectivo valor de dureza Vickers medida como lo presenta la Fig. 8, donde se observa un comportamiento lineal que está adecuadamente descrito en (2) y cuyo coeficiente de correlación de Pearson (R2) es 0,93.

$\mathrm{HV}=-0,0519 \mathrm{P}+18,111$

Donde HV representa la micro dureza Vickers en GPa y P la profundidad máxima perforada. 
Uso de la perforación dinámica como un método alternativo para determinar el comportamiento mecánico de materiales refractarios
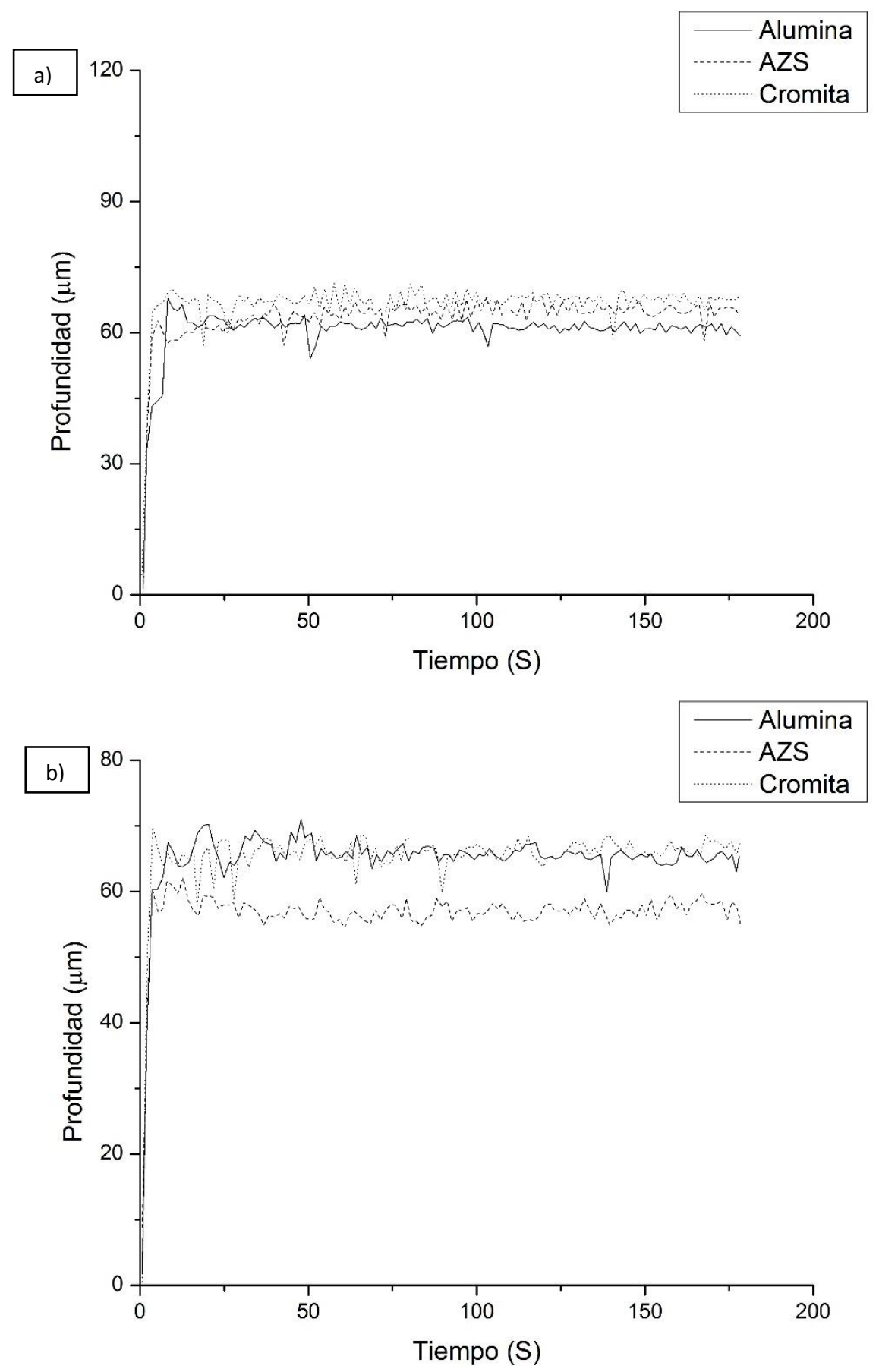

Fig. 6. Curvas profundidad vs tiempo obtenidas durante la perforación con brocas de mampostería: a) Muestras pulidas b) muestras sin pulir, (Fuente: Autores). 
Uso de la perforación dinámica como un método alternativo para determinar el comportamiento mecánico de materiales refractarios
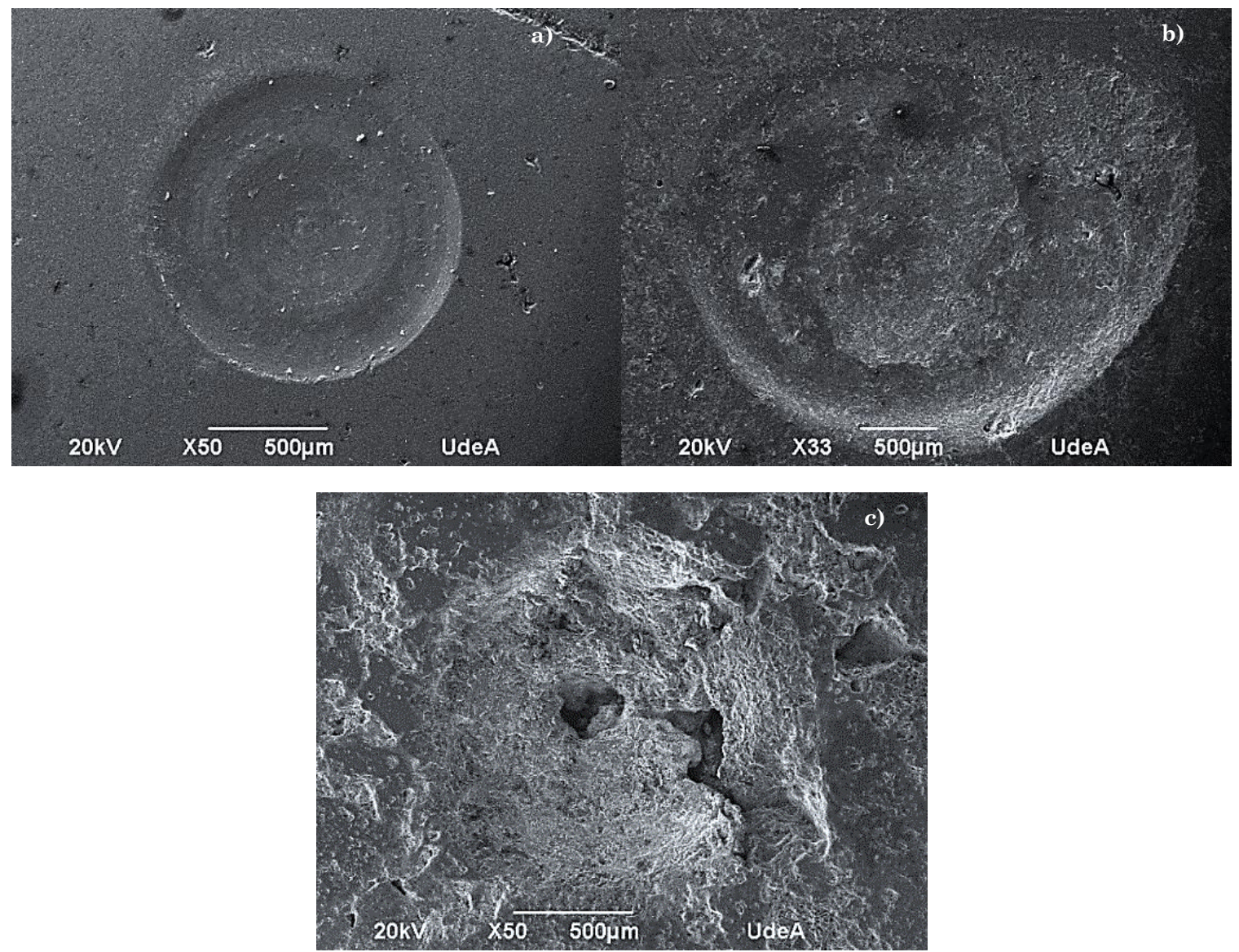

Fig. 7. Morfología de perforaciones realizadas con brocas de mampostería en refractarios de a) alúmina, b) AZS y c) alúmina-cromita. Fuente: Autores.

Tabla 3. Propiedades mecánicas y resultados de perforación de los refractarios estudiados. Fuente: Autores.

\begin{tabular}{lccccc}
\hline \multicolumn{1}{c}{ Material } & $\begin{array}{c}\text { Dureza Vickers } \\
\text { medida }(\mathrm{GPa})\end{array}$ & $\begin{array}{c}\text { Dureza } \\
\text { estimada } \\
(\mathrm{GPa})\end{array}$ & $\begin{array}{c}\text { Dureza } \\
\text { estimada } \\
(\mathrm{GPa})\end{array}$ & $\begin{array}{c}\text { Profundidad máxima } \\
\text { perforada }(\mu \mathrm{m})\end{array}$ & $\begin{array}{c}\text { Desgaste de la broca después } \\
\text { de los ensayos }(\mu \mathrm{m})\end{array}$ \\
\hline Alúmina pulida & $15,10 \pm 1,00$ & 15,27 & 15,22 & $55,60 \pm 11,30$ & $26,00 \pm 12,00$ \\
Alúmina sin pulir & $10,30 \pm 1,00$ & 13,17 & 10,62 & $144,40 \pm 29,10$ & $14,00 \pm 9,00$ \\
\hline AZS pulida & $7,25,00 \pm 18,00$ \\
AZS sin pulir & \multirow{2}{*}{$0,510 \pm 0,02$} & 13,07 & 0,17 & $345,70 \pm 61,60$ & $15,00 \pm 12,00$ \\
\hline Alúmina-cromita pulida & & 2,73 & $296,20 \pm 79,30$ & $31,00 \pm 9,00$ \\
Alúmina-cromita sin pulir & & & & $25,00 \pm 11,00$ \\
\hline
\end{tabular}

1 Valor estimado usando (1).

2 Valor estimado usando (2). 
Uso de la perforación dinámica como un método alternativo para determinar el comportamiento mecánico de materiales refractarios

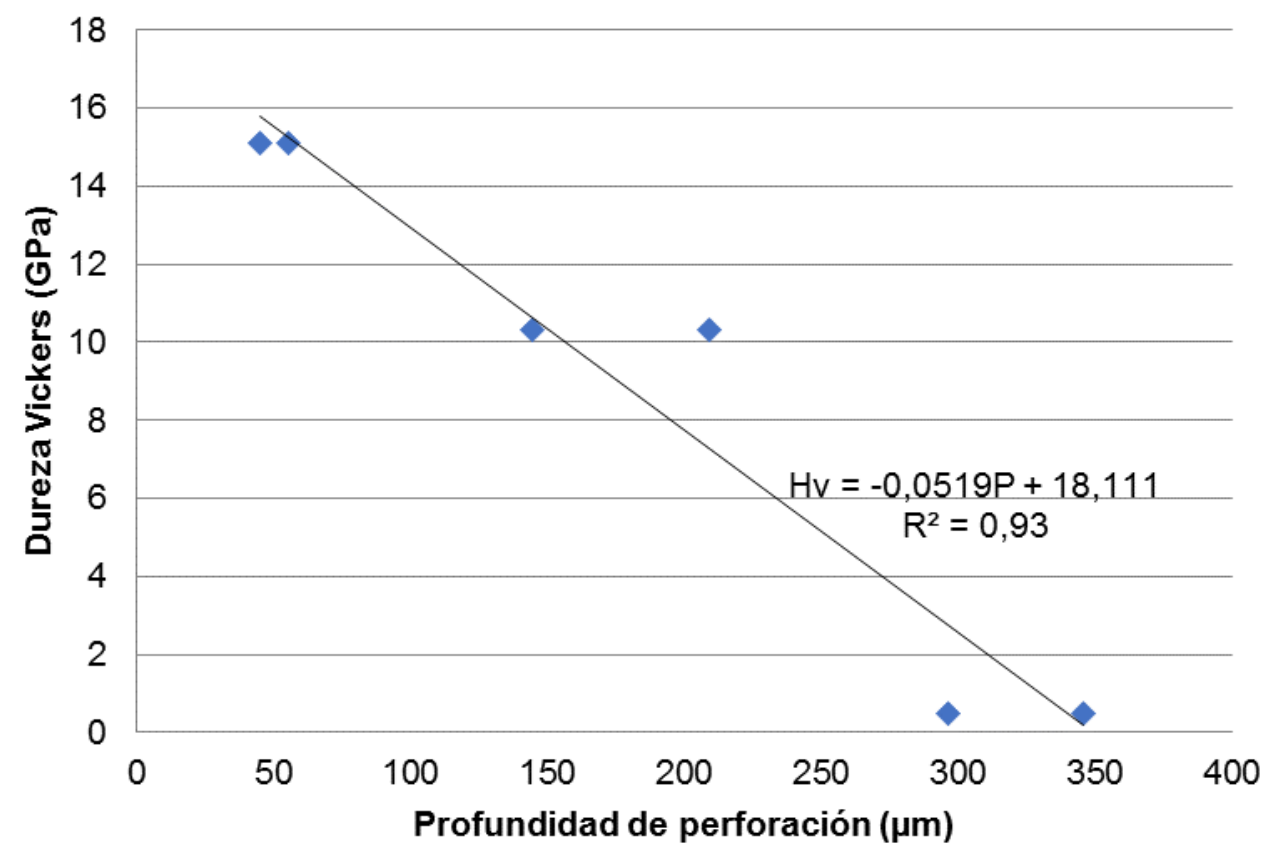

Fig. 8. Correlación entre la dureza Vickers y la profundidad de perforación en los refractarios estudiados. Fuente: Autores.

Lo anterior, indica que la profundidad máxima de perforación, podría ser utilizada para predecir la dureza de refractarios a partir de (2), incluso de aquellos con bajo nivel de densificación, como es el caso del ladrillo de alúmina-cromita, fabricado por prensado y sinterización y en muestras cuya superficie haya sido pulida y sin pulir. La desviación estándar que acompaña a la profundidad máxima perforada en cada muestra es el resultado de factores asociados al contacto entre la broca y la muestras, tales como: la muestra debe ser singular, el desgaste de la broca (ver Tabla 3), la variación en la rugosidad superficial de la muestra evaluada y la presencia de partículas producidas durante el ensayo.

Existen otras expresiones matemáticas que intentan predecir la dureza Vickers de materiales cerámicos como en (1), sin embargo, estas solo alcanzan buenos niveles de correlación con los resultados experimentales para materiales bien consolidados, lo cual en la mayoría de los casos requiere que el material haya sido fundido durante su procesamiento.

\section{CONCLUSIONES}

Los resultados obtenidos a partir de la caracterización mecánica realizada por indentación a los tres refractarios estudiados evidenciaron las dificultades que se presentan para la medida de la dureza en materiales poco densificados y sin una superficie pulida. Así mismo, se hicieron evidentes las limitaciones que tienen algunas expresiones matemáticas que intentan predecir las propiedades mecánicas de materiales cerámicos y cuya aplicación se limita a materiales bien densificados. A pesar de lo anterior, se propone una ecuación que correlaciona adecuadamente la dureza Vickers con la profundidad perforada, tanto en cerámicos densificados como en aquellos que pueden ser considerados friables y la cual mostró tener validez para muestras, tanto pulidas como sin pulir. Por lo anterior, se propone la técnica de perforación con brocas de punta dura como un método alternativo para determinar las propiedades mecánicas de materiales sin las limitaciones existentes para materiales con bajo nivel de consolidación y con superficies rugosas. 


\section{AGRADECIMIENTOS}

Agradecemos al grupo GIMACYR y al fondo de apoyo económico CODI por la financiación del proyecto $\mathrm{PR}$ 15-2-08 y FIT12-1-01.

\section{REFERENCIAS}

[1] C. Barry and G. M. Norton, Ceramic Materials: Science and Engineering. Berlin: Springer, 2013.

[2] R. B. Heimann, Classic and Advanced Ceramics: From Fundamentals to Applications. Weinheim, Germany: WileyVCH Verlag GmbH \& Co. KGaA, 2010.

[3] US. Bureau of Labor Statistics, "Export (Harmonized System): Stone, plaster, cement, asbestos, ceramics, glass etc.," 2016. [Online]. Available: https://research.stlouisfed.org/fred2/series/id xiii. [Accessed: 13-May-2016].

[4] G. D. Quinn, "Indentation Hardness Testing of Ceramics," in ASM Handbook, Mechanical Testing and Evaluation (ASM International), vol. 8, H. K. and D. Medlin, Ed. National Institute of Standards and Technology, 2000, pp. 244-251.

[5] M. Rahman, J. Haider, T. Akter, and M. S. J. Hashmi, "Techniques for Assessing the Properties of Advanced Ceramic Materials," in Comprehensive Materials Processing, Elsevier, Ed. Amsterdam: Elsevier, 2014, pp. $3-34$.

[6] D. Singh, J. Salem, A. Gyekenyesi, and M. Halbig, Mechanical Properties and Performance of Engineering Ceramics and Composites VII, vol. 35, no. 2. Hoboken, NJ, USA: John Wiley \& Sons, Inc., 2012.

[7] I. McColm, Ceramic Hardness. New York: Springer, 1990.

[8] R. C. Bradt, D. Munz, M. SakaiK., and W. White, Fracture Mechanics of Ceramics, Volume 2. Boston, MA: Springer US, 2005.

[9] E. Medvedovski, Ceramic Armor and Armor Systems, 151st ed. Hoboken, NJ, USA: John Wiley \& Sons, Inc., 2006.

[10] Z. Chen, X. Wang, A. Atkinson, and N. Brandon, "Spherical indentation of porous ceramics: Elasticity and hardness," J. Eur. Ceram. Soc., vol. 36, no. 6, pp. 1435-1445, May 2016.

[11] D. Chicot, H. Ageorges, M. Voda, G. Louis, M. A. Ben Dhia, C. C. Palacio, and S. Kossman, "Hardness of thermal sprayed coatings: Relevance of the scale of measurement," Surf. Coatings Technol., vol. 268, pp. 173-179, Apr. 2015.

[12] F. M. Hurtado, A. G. Hernández, M. E. López Gómez, and H. Ageorges, "Estudio de la estructura y las propiedades mecánicas en un recubrimiento de circona estabilizada con $8 \%$ en mol de itria elaborado por proyección térmica por plasma a partir de suspensiones," Matéria (Rio Janeiro), vol. 21, no. 1, pp. 49-60, Mar. 2016.

[13] F. Vargas, "Élaboration de couches céramiques épaisses à structures micrométriques et nanométriques par projection thermiques pour des applications tribologiques.," University of Limoges, 2010.

[14] ASTM, "C1326 - 13 Standard Standard Test Method for Knoop Indentation Hardness of Advanced Ceramics 1," no. March. pp. 1-10, 2003.

[15] ASTM, "C1327 - 15 Standard Standard Test Method for Vickers Indentation Hardness of Advanced Ceramics 1," no. March. pp. 1-10, 2003.

[16] M. Pamplona, M. Kocher, R. Snethlage, and L. A. Barros, "Drilling resistance: overview and outlook [Bohrhärtemessungen: Übersicht und Ausblick]," Zeitschrift der Dtsch. Gesellschaft für Geowissenschaften, vol. 158, no. 3, pp. 665-679, Sep. 2007.

[17] C. Alfes, W. Breit, and P. Schiessl, "Hardness testing for the measurement of stone degradation.," in Proc. 7th Int. Cong. on Deterioration and Conservation of Stone, 1992, pp. 771-780.

[18] J. Delgado Rodrigues and D. Costa, "A new method for data correction in drill resistance tests for the effect of drill bit wear," Int. J. Restor., vol. 10, no. 3, pp. 1-18, 2004.

[19] J. M. Mimoso and D. M. R. Costa, "A new DRMS Drilling Technique for the Laboratory," in International Conference on "Non Destructive Investigations and Micronalysis for the Diagnostics and Conservation of the Cultural and Environmental Heritage., 2005, vol. 8, pp. 113.

[20] E. M. Winkler, Stone: Properties Durability in Man's Environment, vol. 53, no. 9. Vienna: Springer Vienna, 1973.

[21] C. C. Palacio, H. Ageorges, F. Vargas, and A. F. Díaz, "Effect of the mechanical properties on drilling resistance of $\mathrm{Al} 2 \mathrm{O} 3-\mathrm{TiO} 2$ coatings manufactured by atmospheric plasma spraying," Surf. Coatings Technol., vol. 220, pp. 144-148, Apr. 2013.

[22] A. F. Díaz Guarín, "Evolución del desgaste producido mediante perforación mecánica en recubrimientos de alúmina/óxido de titanio obtenidos mediante proyección térmica por plasma, sobre sustratos de aluminio," 
Universidad de Antioquia, 2014.

[23] K. Aristizabal, O. Rojas, F. Vargas, A. F. Díaz, and H. Ageorges, "Efecto del afilado de la broca sobre la resistencia a la perforación de recubrimientos de $\mathrm{AL} 2 \mathrm{O} 3$ - $\mathrm{TiO} 2$ elaborados mediante proyección térmica por plasma," Rev. Colomb. Mater., no. 5, pp. 353360, 2013.

[24] R. E. Chinn, "Image Analysis," in Ceramography Preparation and analysis of ceramic microstructures, Wiley, Ed. New York: Wiley John \& Sons, 2002, pp. 189-198.

[25] "Carbide Masonry Drills." 2015. [online]. Available:

http://www.tatoolsonline.com/uploads/266/saf ety_data_carbide_masonry_tools.pdf. [accessed: 21-sep-2016].

[26] J. Luo and R. Stevens, "Porosity-dependence of elastic moduli and hardness of 3Y-TZP ceramics," Ceram. Int., vol. 25, no. 3, pp. 281-286, Apr. 1999.

[27] K. A. Habib, J. J. Saura, C. Ferrer, M. S. Damra, E. Giménez, and L. Cabedo, "Comparison of flame sprayed $\mathrm{Al} 2 \mathrm{O} 3 / \mathrm{TiO} 2$ coatings: Their microstructure, mechanical properties and tribology behavior," Surf. Coatings Technol., vol. 201, no. 3-4, pp. 1436-1443, Oct. 2006.

[28] J. Lin and J. Duh, "Fracture toughness and hardness of ceria- and yttria-doped tetragonal zirconia ceramics," Mater. Chem. Phys., vol. 78, no. 1, pp. 253-261, Feb. 2003.

[29] K. Maca, M. Trunec, and R. Chmelik, "Processing and properties of fine-grained transparent MgAl2O4 ceramics," Ceram. Silikáty, vol. 51, no. 2, pp. 94-97, 2007.

[30] Matweb.com, "Property values are typical of naturally occurring Quartz. Actual property values will vary from sample to sample." MatWeb.

[31] Artisteer, "AZS Medium density, Technical properties and information." 2015. [online] Available:

http://www.dakot.co.za/index.php/productlist/azs-medium-density. [accessed: 21-sep2016]. 